\title{
Ferric Iron Brain Deposition as the Cause, Source and Originator of Chronic Neurodegenerative Diseases
}

Lucijan Mohorovic $^{1 *}$, Anna Lavezzi ${ }^{2}$, Sanja Stifter ${ }^{3}$, George Perry $^{4}$, Djulija Malatestinic ${ }^{5}$, Vladimir Micovic ${ }^{1}$ and Eris Materljan ${ }^{6}$

${ }^{1}$ Department of Environmental Medicine, School of Medicine, University of Rijeka, Croatia

${ }^{2}$ Department of Biomedical, Surgical and Dental Science, University of Milan, Italy

${ }^{3}$ Department of Pathology, School of Medicine, University of Rijeka, Croatia

${ }^{4}$ College of Science, The University of Texas at San Antonio, USA

${ }^{5}$ Department of Social Medicine and Epidemiology, School of Medicine, University of Rijeka, Croatia

${ }^{6}$ Department of Family Medicine, School of Medicine, University of Rijeka, Croatia

\begin{abstract}
We want to understand the sources of oxidants as factors in understanding the role they play in the pathogenesis of neurodegeneration, such as Alzheimer's disease (AD). Our earlier observations pointed to the difference of intracellular iron when it originates from physiological hemoglobin versus pathological methemoglobin degradation, where heme oxygenation of hemoglobin results in ferrous $\left(\mathrm{Fe}^{2+}\right)$ iron, while methemoglobin catabolism produces ferric $\left(\mathrm{Fe}^{3+}\right)$ iron. Methemoglobin plays the role of carrier, donor and source of cytotoxic redox-active ferric $\left(\mathrm{Fe}^{3+}\right)$ iron and also plays a critical role as an originator of neurodegenerative diseases. Environmental toxicity factors determine a permanent intracellular source of redox-active ferric $\left(\mathrm{Fe}^{3+}\right)$ iron which without ferrous-ferric inversions, 'in situ', has a direct impact on the endothelial small vessels of the brain, increasing the rate of capillary endothelial cell apoptosis and possible cross into brain parenchyma, to astrocytes, glia, neurons, and other neuronal cells, to cause greater degeneration. Understanding the transport and neuronal accumulation of ferric $\left(\mathrm{Fe}^{3+}\right)$ iron, points to how microvessels are organized into a well structured neurovascular unit, with harmful consequences for the brain.
\end{abstract}

Previously our research indicated neonatal jaundice incidence $(p=0.034)$, and heart murmur at a later age $(p=0.011)$, and found that the incidence of children and adults displaying mild disorders such as dyslalia and learning/memory impairments $(p=0.002)$ was significantly higher than in children and adults of mothers lacking methemoglobinemia during pregnancy. Our results point to the consequences of mother-fetal methemoglobinemia caused by environmental oxidants, consequences which not been precisely demonstrated yet. The effects of exogenously induced oxidative stress on the structure and function of the vascular endothelial are direct targets of free hemoglobin and of its oxidative derivative methemoglobin which readily release heme, an abundant source of redox-active iron, and react with sulfur compounds synergy during pregnancy, causing early and late vascular endothelial dysfunction in vital organs and the CNS through 'neurovascular unit' damage, which plays a critical role in understanding oxidative stress in the pathogenesis of neurodegeneration. The harmful effects of mother-fetal pregnancy complications include increased brain non-heme ferric iron deposition, which has been confirmed by the results of histochemical research. According to our hypothesis such a process could result in neuronal death in humans, and with ageing, leading finally to hard neurodegenerative brain diseases such as $A D, P D$ and others.

Keywords: Hemoglobin and methemoglobin catabolism; Brain capillary; Ferric iron; Blood brain barrier; Apoptosis; Neurovascular unit; Neurodegenerative brain disease

\section{Introduction}

It is still not known why redox-active iron levels are abnormally high in some regions of the brain in neurodegenerative disorders, just as it is not clear whether iron accumulation in the brain is an initial event that causes neuronal death or a consequence of the disease process. We looked at the sources of redox-active iron as a key factor in understanding the role of oxidants and oxidative stress and ironinduced oxidative stress as a constitutent and common mechanism involved in the development of the neurodegenerative process in Alzheimer's disease. In the light of previous research we consider that, at least in some neurodegenerative disorders, brain iron misregulation is an initial cause of neuronal death and that this misregulation might be linked to either genetic or non-genetic factors.

Ferric-iron brain accumulation as a cause of neurodegenerative brain disease: A new insight to understanding the mechanism of iron transport

The current view has been derived from research results, with particular attention paid to the role of environmental toxicity factors in brain endothelial small vessels. Our research showed that people continuously inhaling strong oxidants such as $\mathrm{NO}_{\mathrm{x}}\left(\mathrm{NO}\right.$ and $\left.\mathrm{NO}_{2}\right)$ reversibly oxidize oxyhemoglobin $\left(\mathrm{Fe}^{2+}\right)$ to methemoglobin $\left(\mathrm{Fe}^{3+}\right)$.
Irreversible methemoglobinemia can arise because of the disruption of the oxidant/antioxidant balance, supported by synergic $\mathrm{SO}_{2}$ metabolites leading to the degradation of antioxidant thiols [1]. Methemoglobin by itself, and heme, have prooxidant properties and induce structural and functional changes in the vascular endothelium $[2,3]$. These changes include cell growth arrest, senescence, morphological alterations and cell apoptosis, and they lead to both vessel thrombosis, and endothelial cell denudation under the influence of redox-active ferric iron $\left(\mathrm{Fe}^{3+}\right)$, as a product of heme-oxygenase, which is responsible for methemoglobinheme degradation [4]. In the blood, nitric oxide and superoxide form peroxinitrites (ONOO) that convert oxyhemoglobin into methemoglobin, and the methemoglobin-released from heme induces endothelial cytolysis [5]. The toxicity of $\mathrm{H}_{2} \mathrm{O}_{2}$ is also dependent upon

*Corresponding authors: Lucijan Mohorovic, Department of Environmenta Medicine, School of Medicine, University of Rijeka, Creska 2, 52221 Rabac Croatia, Tel: 0915236321 E-mail: lucijan.mohorovic@pu.t-com.hr

Received December 21, 2015; Accepted December 22, 2015; Published December 28, 2015

Citation: Mohorovic L, Lavezzi A, Stifter S, Perry G, Malatestinic D, et al. (2015) Ferric Iron Brain Deposition as the Cause, Source and Originator of Chronic Neurodegenerative Diseases. J Environ Anal Chem 2: 169. doi:10.4172/23802391.1000169

Copyright: (c) 2015 Mohorovic L, et al. This is an open-access article distributed under the terms of the Creative Commons Attribution License, which permits unrestricted use, distribution, and reproduction in any medium, provided the original author and source are credited. 
its reaction with ferrous iron to form hydroxyl radicals by the Fenton reaction. The ferrous iron needed for this reaction is formed by the reduction of cellular ferric iron by superoxide ions [6]. We would like to emphasize the difference between physiological hemoglobin catabolism, and pathological methemoglobin catabolism, because their different final products, ferrous and ferric iron, have distinct characteristics. Ferrous iron has the potential for catalyzing and generating highly cytotoxic hydroxyl radicals as from the Fenton reaction (Ferrous iron $+\mathrm{H}_{2} \mathrm{O}_{2} \rightarrow$ Ferric iron $\left.+\mathrm{OH}+\mathrm{OH}\right)$. Ferric iron is then reduced back to ferrous iron, a peroxide radical and proton by the same hydrogen peroxide. The substantial difference between the intracellular ferric iron originates from the Fenton reaction. Ferric iron originates from methemoglobin catabolism perform a level of methemoglobin cellular uptake which increases the methemoglobinemia leading to Ferric-ironinduced oxidative stress injury. We consider that from methemoglobin catabolism the last product of Ferric iron is a significant added source of ferric iron derived from the Fenton reaction, whose continuious formation has an impact upon the brain neurovascular unit. According to our hypothesis this could be the cause of neuronal death in humans, and of the ageing process, leading finally to hard neurodegenerative disorders such as $\mathrm{AD}, \mathrm{PD}$ and others. Our view confirms the statement that iron and iron-induced oxidative stress constitute a common mechanism that is involved in the development of neurodegeneration. According to the suggestions of previous research we consider that, at least in some neurodegenerative disorders, brain iron misregulation is an initial cause of neuronal death and that this misregulation might be the result of either genetic or non-genetic factors [7]. Our previous research results suggest that methemoglobin plays a particularly important role as carrier and donor of the cytotoxic and redox-active Ferric $\left(\mathrm{Fe}^{3+}\right)$ iron, and determines how iron is transported intracellularly (Figures 1-3). Neuroscience has traditionally focused on the neurons of the central and peripheral nervous systems, and it is now becoming clear that neurons, glia and microvessels are organized into a well structured neurovascular unit, and recent studies have highlighted the importance of brain endothelial cells in this modular organization [8]. Iron is essential for normal cell function, but it also generates toxic reactive oxygen species (ROS) that adversely affects the vascular endothelium of the blood-brain barrier. Human neuroblastoma IMR32 cells' exposure to ferric ammonium citrate(FAC) as a model of neuronal iron overload and neurodegeneration was investigated. In the consequences of the exposure of cells to ferric ammonium citrate it was found that exposure was associated with increased oxidant cell levels, activation of redox-sensitive signals, and apoptosis [9]. Leung et al. have demonstrated that ferric heme is significantly more prooxidant than is ferrous heme. In addition they have shown that this ferric heme has a much higher relaxivity than its ferrous counterparts. In tandem, this evidence suggests that the MR imaging-detected T1 (longitudinal relaxation time) high signal intensity within the vessel wall is an endogenous biomarker of an intraplaque environment that is highly pro-oxidant and proatherogenic. MR imaging measures showed a T1 relaxivity that was 10 times higher for ferric than for ferrous forms of hemoglobin. Their results support the hypothesis that ferric methemoglobin-generated $\mathrm{T} 1$ high signal intensity reflects a prooxidant environment that, in the setting of vessel wall disease, might be proatherogenic [10]. This justifies the study of the oxidant effect of methemoglobin catabolic products on vital organs and the CNS, resulting in their dysfunction. The research MRI data showed that extracellular methemoglobin generates significantly more lipid oxidation than intracellular products; however, methemoglobin in both these environments has similar measures of r1 (longitudinal relaxivity of relaxation rate). Therefore, the $\mathrm{T} 1$ high signal intensity due to methemoglobin is not solely restricted to an environment that causes lipid oxidation. Thus, the ability of this high signal intensity to reflect at-risk plaque may be diminished. However, it is known that in the absence of any chemical modifications, ferric heme substantially degrades the integrity of the RBC membrane, and the eventual fate of a static RBC is lysis. Thus, intracellular methemoglobin is destined to rapidly become extracellular, thereby adding to the oxidative drive [11]. The cellular and intercellular iron transport mechanisms in the central nervous system (CNS) are still poorly understood, meanwhile accumulating evidence suggests that impaired iron metabolism is an initial cause of neurodegeneration [12]. Brar et al. concluded that the development of parkinsonism during the course of $\mathrm{AD}$ appears to be associated with the accumulation of iron, which in turn may contribute to the pathogenesis of neurologic decline [13].

\section{Results}

Our results point out the consequences of brain damage caused by toxic environmental oxidants with a view to the role of methemoglobin catabolism in pregnancy as the source of ferric $\left(\mathrm{Fe}^{3+}\right)$ iron form concentrated in various brain regions. Methemoglobin and hemolysis both occur as a result of oxidative stress, but the prevalent difference

$\mathrm{NO}+$ Superoxide $\left(\mathrm{O}_{2}^{-}\right)$= peroxinitrite $(\mathrm{ONOO})$ a strong oxidant

The erythrocytes are catabolised with the process where ONOO causes intravascular hemolysis

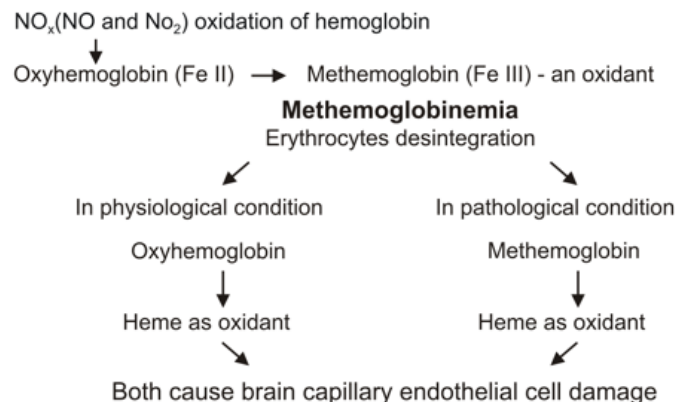

Figure 1: The hemoglobin catabolism.
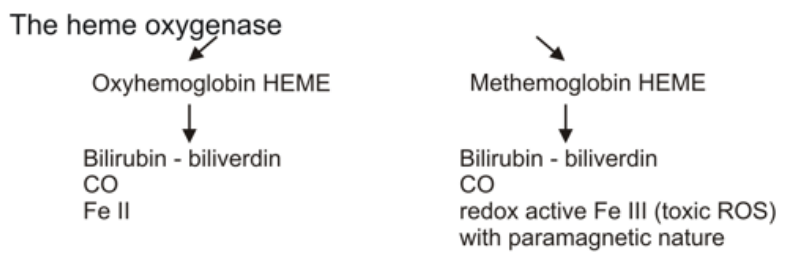

Figure 2: The heme oxygenase[2]

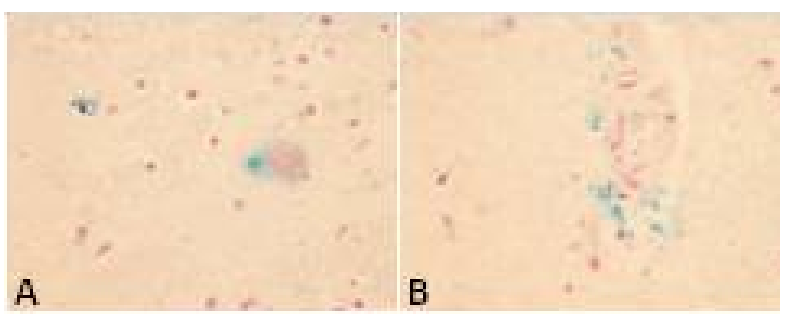

Figure 3: Free iron positivity in the cerebral parenchyma of a 77 year old patient with Alzheimer's disease. A) Focal distribution of positive glia and neurons; B) accumulation of strong iron-positive perivascular regions. Staining: Perl's Prussian Blue reaction for ferric iron-Magnification 40X Source: Stifter Sanja, Department of Pathology, University of Rijeka School of Medicine. 
between them is that methemoglobin is a reversible phenomenon (oxidant-antioxidant balance) whereas hemolysis, which occurs as a result of oxidative stress on the erythrocyte membrane, is an irreversible event. Methemoglobinemia can additionally exacerbate an existing anemia, stimulating hypoxia that may be additionally dangerous. Our prospective study of methemoglobin in pregnancy revealed a significant rise in the level of methemoglobin $>1.5 \mathrm{~g} / \mathrm{L}(r=0.72, p<0.01)$ in the airpolluted exposure period, which can be explained on the basis of an oxidant-antioxidant imbalance, resulting in methemoglobinemia [14]. Methemoglobinemia and stillbirth recorded throughout the exposure period were significantly higher than those recorded in the control period $(p=0.0205)$ and the frequencies of reproductive loss were significantly lower in the control than in the exposure period $(p<0.05)$ [15]. As we have found no evidence of the consequences of mother methemoglobinemia on the fetus, the second objective was to direct attention to methemoglobin as an early biomarker of the oxidative stress effects caused by environmental toxicity, which put pregnancy at risk and may later impair the health of newborns, children and adolescents. Our research found neonatal jaundice incidence $(\mathrm{p}=0.034)$, heart murmur at a later age $(\mathrm{p}=0.011)$, as well as child and adult mild disorders such as dyslalia and learning/memory impairments $(\mathrm{p}=0.002)$ which were significantly higher than in children and adults of control mothers without pregnancy methemoglobinemia [16]. Lavezzi et al. recently presented findings, confirmed with pathohistological techniques, that under adverse conditions, ferric iron positivity in capillary endothelial cells of the blood-brain barrier in the fetus rise, also resulting in preterm birth, stillbirth or early neonatal death [17]. The application of the Blue Prussian method highlighted accumulations of blue granulations, indicative of nonheme $\mathrm{Fe}^{3+}$-positive reactions, in the brainstem and cerebellum of 12 (33\%) of 36 victims and in none of the control group. In the positive cases, iron deposits were widespread in brain parenchyma or localized in specific areas showing a variable extent and intensity (Figure 4 ).

\section{Discussion}

According to our observations, we point out specific cellular methemoglobin and heme catabolism when the last product leading to Ferric iron which will yield cytotoxic and paramagnetic property has an notable role 'in situ'. We propose that ferric iron and ferric ironinduced oxidative stress constitute a common mechanism involved in the development of neurodegeneration, and also sugests an initial cause of neuronal death as a result of environmental toxicity factors. The experiments showed that ferric and ferrous iron can enter cells via different pathways, they do not indicate which pathway is dominant in humans [18]. Heme, the major functional form of iron, is synthesized in the mitochondria. Smith et al. suggest that iron is able to participate in 'in situ' oxidation and readily catalyzes an $\mathrm{H}_{2} \mathrm{O}_{2}$-dependent oxidation, and indicates that iron accumulation could be an important contributor toward the oxidative damage of Alzheimer's disease [19]. Our work, according to our standpoint, supports the above statement about the importance of disturbed heme metabolism when the heme oxygenase-1, an enzyme that catalyzes the conversion of methemoglobin and heme to ferric iron, is increased in Alzheimer's disease suggesting increased heme turnover as a source of redox-active iron. Perry et al. have found that while mitochondrial DNA and cytochrome $\mathrm{C}$ oxidase activity are increased in Alzheimer's disease, the number of mitochondria is decreased, indicating accelerated mitochondria turnover, and suggesting mitochondrial dysfunction as a potentially inseparable component of the initiation and progression of Alzheimer's disease [20]. It was also found that oxidative damage occurs primarily within the cytoplasm rather than in mitochondria. According to this

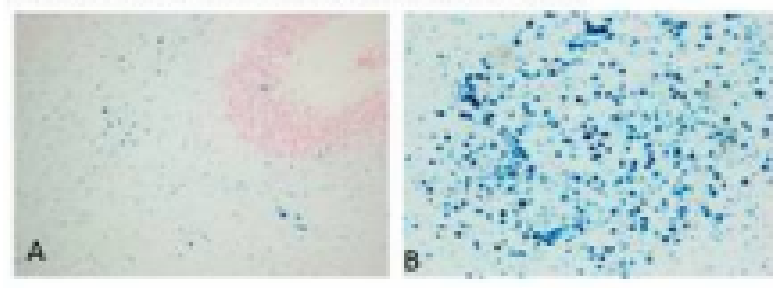

Figure 4: Free iron positivity in the subcortical region $(A)$ and parenchyma (B) of the cerebellum of a 6 month-old victim of SIDS. Staining: Perl's Prussian Blue reaction for ferric-iron-Magn. 20X. Source: Lavezzi AM, Mohorovic L Alfonsi G, Coma MF, Matturri L, Brain iron accumulation in unexplained fetal and infant death victims with smoker mothers. The possible involvement of maternal methemoglobinemia. Lino Rossi Research Center for the study and prevention of unexpected perinatal death and SIDS, Department of Surgical, Reconstructive and Diagnostic Sciences, University of Milan, Italy.

hypothesis that mitochondria acts as a source of hydrogen peroxide, an intermediate, once in the cytoplasm, will be converted into highly reactive hydroxyl radicals through the Fenton reaction in the presence of metal ions (iron and copper) causing damage to the cytoplasm [21]. Cell apoptosis is initiated by extracellular and intracellular signaling pathways, the death receptor- and the mitochondria-mediated pathway. Various pathologies can result from oxidative stress-induced apoptotic signaling consequently leading to ROS increases and/or antioxidant decreases, disruption of intracellular redox homeostasis, and irreversible oxidative modifications of lipid, protein, or DNA [22]. Furthermore, iron participates in diverse pathologic processes by the Fenton reaction, which catalyzes the formation of reactive oxygen species (ROS). To test the hypothesis that this reaction accelerates apoptosis, Jacob et al. used human umbilical vein endothelial cells (HUVECs) as surrogates for the microvasculature in vivo. HUVECs were loaded with $\mathrm{Fe}^{3+}$ (ferric chloride and ferric ammonium citrate), and apoptosis executed after a heat shock stimulus [23]. Brain iron is a major contributor to magnetic resonance imaging (MRI) contrast in normal gray matter. Non-heme brain iron is present mainly in the form of ferritin. The quantitation of non-heme brain ferric iron indicated by MRI helps in the diagnosis and monitoring of different neurological diseases [24]. Most of the brain non-heme iron is believed to be present as a storage pool consisting of ferritin or hemosiderin and also as a product of methemoglobin catabolism [25]. However, the concentration of transferin-bound iron is always far too small to affect MRI. This fact suggests considering the role of methemoglobin catabolism as the source of ferric iron $\left(\mathrm{Fe}^{3+}\right)$ form concentrated in various brain regions. Nowdays, non-heme-bound $\mathrm{Fe}^{3+}$ is quantified using Magnetic Resonance Imaging (MRI), thanks to its paramagnetic properties. It is believed that most non-heme-bound iron is deposited in the form of ferritin, haemosiderin, or methemoglobin catabolic products, whereas transferrin-bound iron concentration is always low and can not be detected by MRI [26]. Recent research results indicate a ferrihydrite-magnetite core-shell ferritin structure. It was also found that the magnetite in the ferritin iron core is not a source of free toxic ferrous iron, as previously believed. Therefore, the presence of magnetite in the ferritin cores of patients with Alzheimer's disease is not a cause of their increased brain ferrous iron(II) concentration [27].

\section{Conclusion}

Methemoglobin and heme have prooxidant properties. Abundant in the source of cytotoxic and redox-active ferric $\left(\mathrm{Fe}^{3+}\right)$ iron which without ferrous-ferric inversions, 'in situ' as a cause of iron -induced oxidative stress, have an direct and specific impact on the brain 
Citation: Mohorovic L, Lavezzi A, Stifter S, Perry G, Malatestinic D, et al. (2015) Ferric Iron Brain Deposition as the Cause, Source and Originator of Chronic Neurodegenerative Diseases. J Environ Anal Chem 2: 169. doi:10.4172/2380-2391.1000169

endothelial small vessels, and increase the rate of endothelial cell apoptosis and so make possible the accumulation of methemoglobin, heme and ferric iron, in brain parenchyma. Our results identify the consequences of mother-fetal methemoglobinemia caused by environmental oxidants. Under the gradual influence of free radicals on physiological erythrocytes and pathological methemoglobin degradation, we found significant incidence of neonatal bilirubinemia, heart murmur and learning/memory impairments in childhood and teenagers, which has not been precisely demonstrated yet. In conclusion we point out the importance of methemoglobinemia not only as the biomarker and precursor of the effects of environmental oxidants, but a carrier and donor of redox-active ferric iron. We identify ferric iron as an originator having an important role in crossing brain microvessels to neurons (neurovascular unit), causing neuronal death and continous ageing process, and leading finally to hard neurodegenerative disorders such as $\mathrm{AD}, \mathrm{PD}, \mathrm{MS}$ and other diseases. Nevertheless our findings as to the relation between environmental oxidants and the pathogenesis of neurodegenerative diseases need further research.

\section{References}

1. Mohorovic L, Materljan E, Micovic V, Malatestinic D, Stifter S, et al. (2012) Impacts of Exogenously Derived Nitrogen Oxide and Sulfur Compounds on the Structure and Function of the Vascular Endothelium Link Pregnancy Hypertension with Later Life Hypertension. J Hypertens Open Access.

2. Balla J, Vercellotti GM, Jeney V, Yachie A, Varga Z, et al. (2007) Heme, heme oxygenase, and ferritin: how the vascular endothelium survives (and dies) in an iron-rich environment. Antioxid Redox Signal 9: 2119-2137.

3. Jeney V, Balla J, Yachie A, Varga Z, Vercellotti GM, et al. (2002) Pro-oxidant and cytotoxic effects of circulating heme. Blood 100: 879-887.

4. Maisels MJ, Kring E (2006) The contribution of hemolysis to early jaundice in normal newborns. Pediatrics 118: 276-279.

5. Denicola A, Souza JM, Radi R (1998) Diffusion of peroxynitrite across erythrocyte membranes. Proc Natl Acad Sci 95: 3566-3571.

6. Starke PE, Farber JL (1985) Ferric iron and superoxide ions are required for the killing of cultured hepatocytes by hydrogen peroxide. Evidence for the participation of hydroxyl radicals formed by an iron-catalyzed Haber-Weiss reaction. J Biol Chem 260: 10099-10104.

7. Qian ZM, Shen X (2001) Brain iron transport and neurodegeneration. Trends Mol Med 7: 103-108.

8. Abbott NJ, Rönnbäck L, Hansson E (2006) Astrocyte-endothelial interactions a the blood-brain barrier. Nat Rev Neurosci 7: 41-53.

9. Salvador GA, Oteiza PI (2011) Iron overload triggers redox-sensitive signals in human IMR-32 neuroblastoma cells. Neurotoxicology 32: 75-82.

10. Leung G, Moody AR (2010) MR imaging depicts oxidative stress induced by methemoglobin. Radiology 257: 470-476.

11. Baysal E, Monteiro HP, Sullivan SG, Stern A (1990) Desferrioxamine protects human red blood cells from hemin-induced hemolysis. Free Radic Biol Med 9: 5-10.

12. Mills $E$, Dong XP, Wang F, Xu H (2010) Mechanisms of brain iron transport: insight into neurodegeneration and CNS disorders. Future Med Chem 2: 51-64.
13. Brar S, Henderson D, Schenck J, Zimmerman EA (2009) Iron accumulation in the substantia nigra of patients with Alzheimer disease and parkinsonism. Arch Neurol 66: 371-374.

14. Mohorovic $L$ (2003) The level of maternal methemoglobin during pregnancy in an air-polluted environment. Environ Health Perspect 111: 1902-1905.

15. Mohorovic L, Micovic V (2012)The importance of 'first-blood circulation stage',a new insight into the pathogenesis of clinical manifestations of preeclampsia. Advances in Bioscience and Biotechnology 3: 945-950.

16. Mohorovic L, Materljan E, Brumini G (2010) Consequences of methemoglobinemia in pregnancy in newborns, children, and adults: issues raised by new findings on methemoglobin catabolism. J Matern Fetal Neonatal Med 23: 956-959.

17. Lavezzi AM, Mohorovic L, Alfonsi G, Corna MF, Matturri L (2011) Brain iron accumulation in unexplained fetal and infant death victims with smoker mothers--the possible involvement of maternal methemoglobinemia. BMC Pediatr 11: 62.

18. Conrad ME, Umbreit JN, Moore EG, Hainsworth LN, Porubcin M, et al. (2000) Separate pathways for cellular uptake of ferric and ferrous iron. Am J Physio Gastrointest Liver Physiol 279: G767-774.

19. Smith MA, Harris PL, Sayre LM, Perry G (1997) Iron accumulation in Alzheimer disease is a source of redox-generated free radicals. Proc Natl Acad Sci USA 94: 9866-9868.

20. Perry G, Taddeo MA, Petersen RB, Castellani RJ, Harris PL, et al. (2003) Adventiously-bound redox active iron and copper are at the center of oxidative damage in Alzheimer disease. Biometals 16: 77-81.

21. Marlatt M, Lee HG, Perry G, Smith MA, Zhu X (2004) Sources and mechanisms of cytoplasmic oxidative damage in Alzheimer's disease. Acta Neurobiol Exp (Wars) 64: 81-87.

22. Circu ML, Aw TY (2010) Reactive oxygen species, cellular redox systems, and apoptosis. Free Radic Biol Med 48: 749-762.

23. Jacob AK, Hotchkiss RS, DeMeester SL, Hiramatsu M, Karl IE, et al. (1997) Endothelial cell apoptosis is accelerated by inorganic iron and heat via an oxygen radical dependent mechanism. Surgery 122: 243-253

24. Vymazal J, Brooks RA, Patronas N, Hajek M, Bulte JW, et al. (1995) Magnetic resonance imaging of brain iron in health and disease. J Neurol Sci 134 Suppl: $19-26$

25. Schenck JF, Zimmerman EA (2004) High-field magnetic resonance imaging of brain iron: birth of a biomarker? NMR Biomed 17: 433-445.

26. Rouault TA, Cooperman S (2006) Brain iron metabolism. Semin Pediatr Neurol 13: $142-148$.

27. Gálvez N, Fernández B, Sánchez P, Cuesta R, Ceolín M, et al. (2008) Comparative structural and chemical studies of ferritin cores with gradual removal of their iron contents. J Am Chem Soc 130: 8062-8068. 